\title{
Computer Simulation of Power Deviation, Admittance Distance, Coherency Indices, and Dynamic Equivalents for Electric Power Systems
}

\author{
Ben C. Ubah \\ Europa-Universität Viadrina, \\ Frankfurt (Oder)
}

\author{
Kelechi Azubuike \\ 4328 Brisebois Drive, NW, Calgary, Alberta, \\ Canada
}

\begin{abstract}
This paper presents a computer simulation of coherencybased dynamic equivalents for the reduction of large power systems for faster transient stability studies applicable in academic and research fields. The simulation decomposes the power system generators into the study and external areas by computing the power deviation of all generators during a fault. The simulation features the computation of admittance distance, inertia and damping indices for the identification of coherent machines, and dynamic equivalents. The simulation tool comprises a set of scripts that were developed on the MATLAB platform and are easily operated through the use of the Command window. The theoretical formulations of the algorithms implemented by this simulation are described while its computational extensiveness is tested with the IEEE 10 generator 39-bus system and the Nigeria power system. The illustrative approach in this paper provides a more practical approach to dynamic equivalents based on coherent generators by expounding relevant theoretical and computational ideas.
\end{abstract}

\section{General Terms}

Coherency-based dynamic equivalents. Simulation, Power System analysis, Stability studies

\section{Keywords}

Coherency, dynamic equivalents, electrical coupling, Nigeria power system simulation, transient stability analysis, MATLAB

\section{INTRODUCTION}

An efficient and reliable electrical power system network remains a main key to modernization. However, due to the increasing demand of electric power from heavy industrial machineries, home and office appliances, the power system continues to increase both in size and complexity. This in turn has created analytical challenges in the operation and planning of power systems.

The electrical power system is not an exception to the impact of advancement of digital computer in design and simulation of engineering systems. In recent times, the computer has been used to develop a variety of advanced tools that can be applied to the simulation of large power systems.

The basic intent of computer-based simulation for power system analysis is to reproduce near real phenomena through power system software packages as an efficient solution for academic and research purposes. Software packages have fallen into two classes: commercial software and educational/research-aimed software [1]. Commercial software tools have proven to be computationally efficient and complete but not flexible. For research purposes, the flexibility and the ability of easy prototyping are often more crucial aspects than computational efficiency [1]. Several examples of both commercial and education/research-based software tools have been outlined in [1].

Amongst a host of high level scientific languages, MATLAB has proven to be the best user choice, although, other languages can lead to good results in the field of power systems analysis, MATLAB has gained wide acceptance because of its excellent graphing capabilities, toolboxes and matrix-oriented programming approach $[1,2]$.

With the availability of digital techniques to solve power system problems, the timely, economic and efficient transient stability analysis of large power systems poses a challenge when trying to approximate the dynamic behavior of largescale power systems to further reduce computing effort and resources. This approximation seems inevitable because the increase and continuous interconnections between electrical utilities for both economic reasons and resource sharing has initiated an increase in the conceptual and numerical difficulties experienced while performing system stability calculations. Through construction of dynamic equivalents (DE), the number of differential equations necessary for describing the system becomes less than that of the full system [14]. Thus, such a study through equivalent models simplifies analysis.

This paper describes a MATLAB-based power system simulation for DEs based on coherency for academic and research purposes. The concept of equal velocity and equal acceleration as described by [3] was used to develop the coherency and aggregation algorithms. This simulation program computes the electrical power deviation of all machines during fault, the admittance distance between generators, coherency indices (i.e. inertia and damping conditions) and dynamic equivalents. The program is operated through the Character User Interface (CUI) of MATLAB the Command window - that allows for user interactivity. The results of simulation processes are also displayed on this command line interface; graphical plots are automatically generated when necessary. This software was tested with both the IEEE 10 generator test case and the Nigeria power system and the results are discussed. DE simulations depend on power flow and transient stability results and therefore, these results were obtained using the program described in [6]. The text in [6] contains program files for power system analysis, but does not contain any provision for DEs. The simulation described in this paper is easily integrated with the programs in [6] (which is a quite popular text among undergraduates and first year graduates of power systems). Also, the mathematical formulations used for this simulation were 
obtained from [3] (which did not provide simulation hints) and these are not easily accessible in many power system analysis textbooks. Therefore, the computing approach used in this paper, would go on to inspire better academic learning and research among both undergraduate and graduate students in this area of coherence-based dynamic equivalents, by providing fundamental theoretical and simulation ideas. These ideas are presented in the sections that follow.

\section{THEORITICAL FORMULATION 2.1 Transient Stability Analysis (TSA)}

Power system transient stability concerns the behavioral response of the power system after a large disturbance causes a change in system conditions. Transient stability analysis is computationally intensive as it tries to obtain the electromechanical response of the system [7]. Such analysis concerns the transient behaviour of the power system when moving from pre to post - contingency operating point [7]. Upon the occurrence of large disturbances, the system is moved from its normal operating point and will settle at a new operating point depending on system dynamics.

Multi-machine TSA usually requires solving a set of nonlinear differential equations which increases with increase in the size of the power system. Therefore, to reduce the number of equations that would be solved and also reduce computation time and effort, dynamic equivalents are employed.

\subsection{DEs based on Coherency}

DEs studies go back to 1960's [14] and use various approaches. DEs are, apart from special cases, reduced order models, i.e., the number of differential equations necessary for describing the system is less than of the full system [13]. These approaches include principally two techniques to determine the group of generators to be aggregated [14]. One of them is the modal equivalent technique and the other is the coherency technique [3,13 and 14].

The research proposal in [13] supported the use of modal analysis for dynamic equivalency, by trying to prove that modal techniques are more favourable in connection with controller design techniques; stressing that fact that the technique is based on modes of the system which are independent from excitation, i.e., disturbances are not significant hereby. They further unveil the limitation of the coherency technique by revealing the fact that the method is fault dependent, i.e., for different fault locations, different coherent group of machines emerge. But, this proposal agrees that the coherency technique is the most widely accepted.

However, the proposal in [14] elucidates that dynamic equivalency studies using modal analysis are based on state space representation and faces the obstacle of the need for solving the eigenvalue problem when applying this approach to large systems with hundreds of generating units when the models of each unit may have 10 to 20 state variables. The proposal here was the coherency technique.

In [3] the technique based on the principle of coherency for dynamic equivalency is flagged because of its computational attractiveness and its possession of the virtue to obtain reduced order models that retain the structure (physical correspondence) of the larger power system. This is the technique adopted in this simulation. The technique adopted is based on the concept of equal velocity and acceleration for coherent generators taking into account the effect of damping and transfer conductance in the power system model.

\section{MATHEMATICAL FORMULATION 3.1 Generator Power Deviation (GPD)}

The concept of power deviation of the generators at the instant of initiating fault is applied to classify the machines into study and external (remote) areas. The generators with power deviation less than $30 \%$ are grouped together as the remote area, while the rest, the study system [3]. Coherent groups are identified among only the remote area generators and each coherent group is replaced by a single equivalent machine.

We applied a simple technique of acquiring the power deviation of the generators which involves the use of the reduced admittance matrix, $\boldsymbol{Y}_{\text {red }}$ (for pre-fault and fault-on conditions) obtained after including the transient reactance of the generators and the internal voltages in the network's performance equations and eliminating all load buses by applying Kron's reduction method on the modified system's bus admittance matrix [11].

The equations for current injections at the internal buses can be obtained as:

$$
\left[\begin{array}{c}
0 \\
i_{m}
\end{array}\right]=\left[\begin{array}{ll}
y_{n n} & y_{n m} \\
y_{n m}^{t} & y_{m m}
\end{array}\right]\left[\begin{array}{c}
v_{n} \\
e_{m}^{\prime}
\end{array}\right]
$$

The subscript $m$ denotes the generator buses that are to be retained, and $n$ denotes the load buses that are to be eliminated.

From Eqn. (1) $i_{m}$ can be calculated as:

$$
\begin{aligned}
& i_{m}=\left[y_{m m}-y_{n m}^{t} y_{n n}{ }^{-1} y_{n m} e_{m}^{\prime}\right] \\
& \text { Where, } \\
& \boldsymbol{Y}_{\text {red }}=y_{m m}-y_{n m}^{t} y_{n n}{ }^{-1} y_{n m} e_{m}^{\prime}
\end{aligned}
$$

is the reduced admittance matrix of the system.

The electrical output power of each generator with its internal voltage, $E_{g e n}$ is calculated by first calculating the generator current from eqn. 4 below:

$$
\left[\boldsymbol{I}_{\text {gen }}\right]=\left[\boldsymbol{Y}_{\text {red }}\right]\left[\boldsymbol{E}_{\text {gen }}\right]
$$

Then taking real part of the product

$$
\boldsymbol{P}_{\text {gen }}=\mathfrak{R}\left(\boldsymbol{E}_{\text {gen }} \boldsymbol{I}_{\text {gen }}{ }^{*}\right)
$$

This can be done for the three states of $\boldsymbol{Y}_{\text {red }}$ (i.e., pre-fault, fault - on, and post-fault). Therefore, power deviation (or variation) at the $i_{t h}$ machine,

$$
\Delta P_{e i}=P_{e i}^{p}-P_{e i}^{f}
$$

Where, $P_{e i}^{p}$ and $P_{e i}^{f}$ are the pre-fault and fault-on real powers respectively.

It has been observed by illustrative examples that generators close to a fault location experience greater power deviation than those that are distant. See section 4.1.1 for illustrations by simulation.

\subsection{Admittance Distance Index (ADI)}

Admittance distance (or electrical proximity) is simply a measure of estimating generators' distance (or closeness) to one another using the reduced admittance matrix. Usually, electrically close generators have been observed to cohere in swinging, although this would further depend on other network and machine characteristics which have not been taken into account within the scope of this work. 
As the mutual admittance between two machines become smaller, that pair of machines will be considered more distant to each other and vice versa. Hence, the mutual admittance between a pair of machines can be said to be measure of coupling between them.

From the formulation in [3] it can be deduced that if all the machines have equal mutual admittances to the $i^{\text {th }}$ machine, then

$$
\left|Y^{\prime} i i\right|=(n-1)\left|Y^{\prime} i j\right|
$$

Hence the admittance distance index $\alpha_{i k}$, can be defined by the relation.

$$
\alpha_{i k}=\frac{(n-1)\left|Y^{\prime} i k\right|}{\left|Y^{\prime} i i\right|} \geq 1.0 \text { for } k=1 \ldots n \quad k \neq i
$$

The machines which satisfy Eqn. (8) are considered electrically close enough to be candidates for coherency grouping test with the $i^{\text {th }}$ machine. See section 4.1.2 for illustrative examples.

\subsection{Coherency Indices (CI) - Inertia and Damping}

The concept of equal velocity and acceleration as depicted in [3] was applied in the development of the algorithm for this simulation. A summary of this is shown in this section. The swing equation is being used as a tool for computation of rotor speed. The swing equation of the $i$ th generator with damping included is:

$$
M_{i} \frac{d^{2} \delta i}{d t}=P_{m i}-P_{e i}-D_{i} \omega_{\mathrm{i}}
$$

From the formulation in [3] it was obtained that the linearized acceleration equation of the $i$ th and $j$ th machines are:

$$
\begin{aligned}
& \Delta \ddot{\delta}_{l}= \\
& M_{i}^{-1} Y_{i j}\left(\Delta \delta_{j}-\Delta \delta_{i}\right)+M^{-1} \sum_{\substack{k=1 \\
k \neq i, j}}^{n} Y_{i k}\left(\Delta \delta_{k}-\right. \\
& \left.\Delta \delta_{i}\right)-M_{i}^{-1} D_{i} \Delta \omega_{i} \\
& \Delta \ddot{\delta}_{j}= \\
& M_{j}^{-1} Y_{j i}\left(\Delta \delta_{i}-\Delta \delta_{j}\right)+M^{-1} \sum_{\substack{k=1 \\
k \neq i, j}}^{n} Y_{j k}\left(\Delta \delta_{k}-\right. \\
& \left.\Delta \delta_{j}\right)-M_{j}^{-1} D_{j} \Delta \omega_{j} \\
& \quad \text { where, } Y_{i j}=Y_{j i}
\end{aligned}
$$

For perfect coherency, the third term in the two equations are equal each other, thus, eqn. 13.

$$
M_{i}^{-1} D_{i}=M_{j}^{-1} D_{j}
$$

Details on the derivation of $\beta$ are given in [3].

For coherency, two normalized coherency indices $\beta$ (inertia) and $\gamma$ (damping) are defined as:

and

$$
\beta_{i j}=\frac{\left|M_{i}-M_{j}\right|}{\operatorname{Max}\left(M_{i}, M_{j}\right)}
$$

$$
\gamma_{i j}=\frac{\left|M_{i}^{-1} D_{i}-M_{j}^{-1} D_{j}\right|}{\operatorname{Max}\left(M_{i}^{-1} D_{i}, M_{j}^{-1} D_{j}\right)}
$$

\subsubsection{Coherency Criteria}

Extensive numerical study have shown that a tolerance of $\beta_{i j} \leq 0.5$ and for $\gamma_{i j} \leq 0.5$ give results which are in conformity with direct simulation [3]. Coherent machines will next be aggregated to form single equivalents.

\subsection{Computation of DEs}

Classically, since generators are represented by a voltage behind transient reactance and a coherent pair are connected in parallel (when fictitious points inserted between the voltage sources and the transient reactance of the coherent group are assumed to be connected together) [10], the parallel voltage sources are replaced by a single equivalent voltage source such that it can deliver the active and reactive power equal to the sum of the active and reactive power delivered by the machines of the coherent group being replaced [3]. The simulation algorithm for dynamic equivalents is as described in [3].

Sum of the powers of a group of coherent machines are expressed as in:

$$
\begin{aligned}
& S_{T}=\sum_{i=1}^{m} E_{i}^{*} I_{i} \\
& \text { Where, } E_{i}=V_{i}+X_{d i}^{\prime} I_{i}
\end{aligned}
$$

$V_{i}=$ the $i^{\text {th }}$ generator's terminal voltage and

$I_{i}=$ current flowing to the terminal bus of the $i^{\text {th }}$ generator.

The power delivered by the equivalent machine is:

$$
S_{e}=E_{e}^{*} I_{e}
$$

As stated above, for power equivalence:

$$
\begin{gathered}
S_{T}=S_{E} \\
\text { i.e., } E_{e}^{*} I_{e}=\sum_{i=1}^{m} E_{i}^{*} I_{i} \\
\text { Where, } E_{e}=\left[\sum_{i=1}^{M} E_{i}^{*} \frac{I_{i}}{I_{e}}\right]^{*}
\end{gathered}
$$

and

$I_{e}=\sum_{i=1}^{m} I_{i}=$ Vector sum of currents of the coherent group being replaced

$E_{e}=$ the equivalent generator's voltage

$m=$ Number of generators in the coherent group

The simulation algorithm applied a technique that uses the concept of power invariance at the internal bus, as well as at the terminal buses as expounded in [3]. This techniques leads to a modification of the transient reactance connected from the equivalent internal bus to the network terminal buses.

Hence, for a coherent group containing $m$ number of terminal buses:

The modified transient reactance of the equivalent machine can be computed using Eqn. (22).

$$
\begin{aligned}
& X_{d e}^{\prime}=\frac{\left(\mathrm{E}_{\mathrm{e}}-\mathrm{V}_{\mathrm{e}}\right)}{\sum_{\mathrm{i}=1}^{\mathrm{m}} \mathrm{I}_{\mathrm{i}}} \\
& \text { Where } V_{e}=\left[\sum_{i=1}^{m} \frac{V_{i}^{*} I_{i}}{I_{T}}\right]^{*}
\end{aligned}
$$

$I_{T}=I_{e}$ is the vector sum of current injected at the equivalent terminal bus from the equivalent machine replacing the coherent group of machines

The inertia constant of the equivalent machine is obtained as:

$$
H_{e}=\sum_{i=1}^{m} H_{i}
$$

(referred to a common MVA base if power is in per unit, since $\mathrm{H}$ varies inversely as the MVA base)

The damping coefficient and mechanical power of the equivalent machine are obtained respectively as:

$$
D_{e}=\sum_{i=1}^{m} D_{i}
$$

where $D_{e}=$ Equivalent damping coefficient of the group

$$
\text { Also, } P_{m e}=\sum_{i=1}^{m} P_{m i}
$$

where $P_{m e}=$ mechanical power of the equivalent machine.

\section{Simulation Results and Discussion}

Based on the formulations above, MATLAB scripts were generated to obtain the power deviation after the occurrence of a fault, the admittance distance index, coherency indices and dynamic equivalents. These scripts depend on obtaining the power flow and transient stability variables using the scripts of [6]. 
The IEEE 39 bus, 10 machine test system and the Nigeria 27bus, $330 \mathrm{kV}$ power system were used to test our simulation program. The single-line diagram of the IEEE test system is depicted in Figure 1. The test data was obtained from [3] and the network diagram from [12]. The results of the simulation are described subsequently.

\subsection{Computation of Coherency Parameters}

\subsubsection{GPD}

A MATLAB script (powerVar.m) was written to simulate the power deviation of all generators. With this script in the Current Directory of MATLAB, the power deviation simulation is carried out by typing the following command on the terminal window of MATLAB:

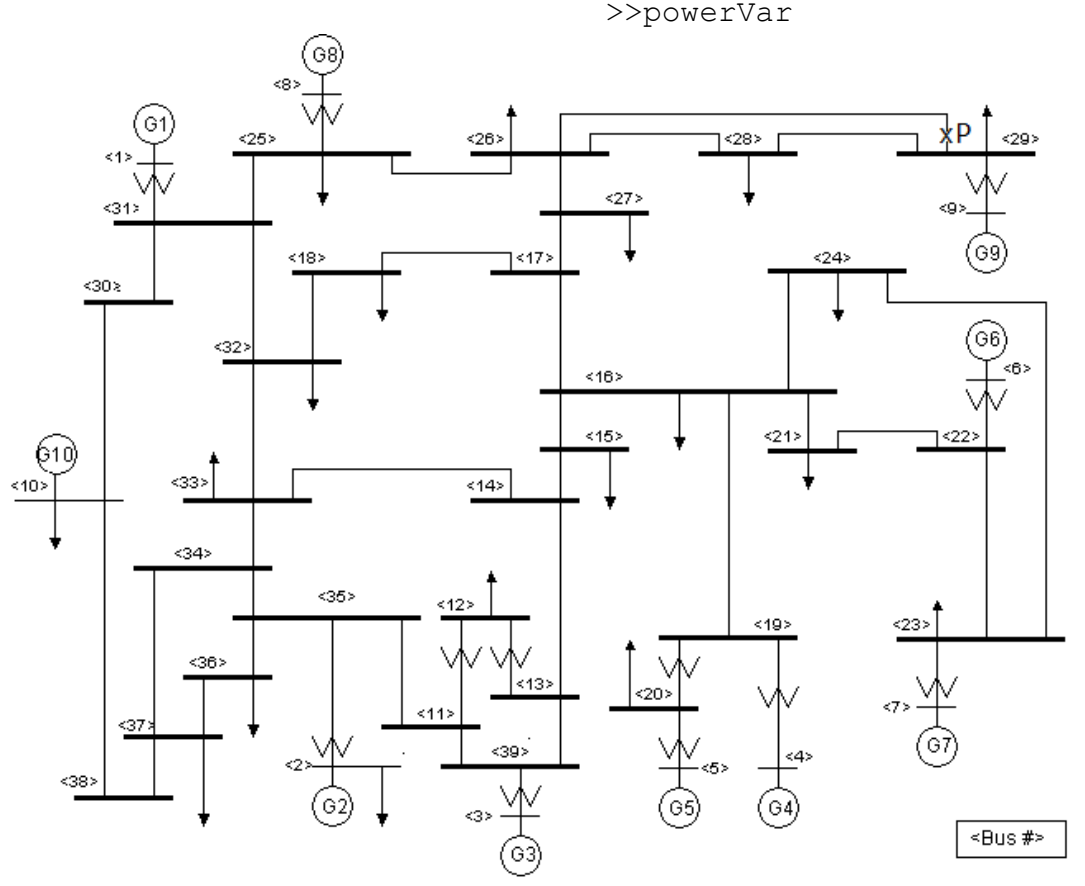

Figure 1: IEEE 10 generator 39-bus system

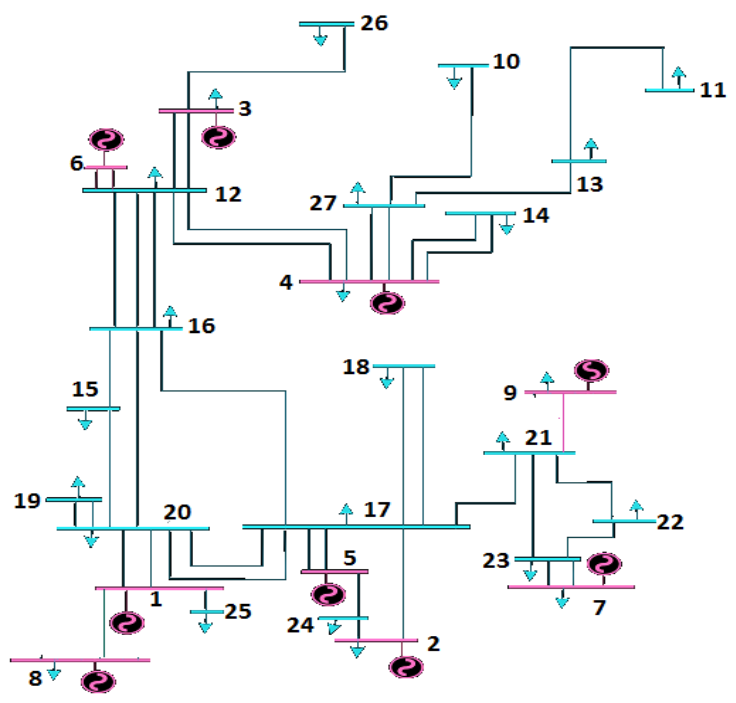

Figure 2: Single line diagram of the Nigerian $330 \mathrm{kV}$ power system 


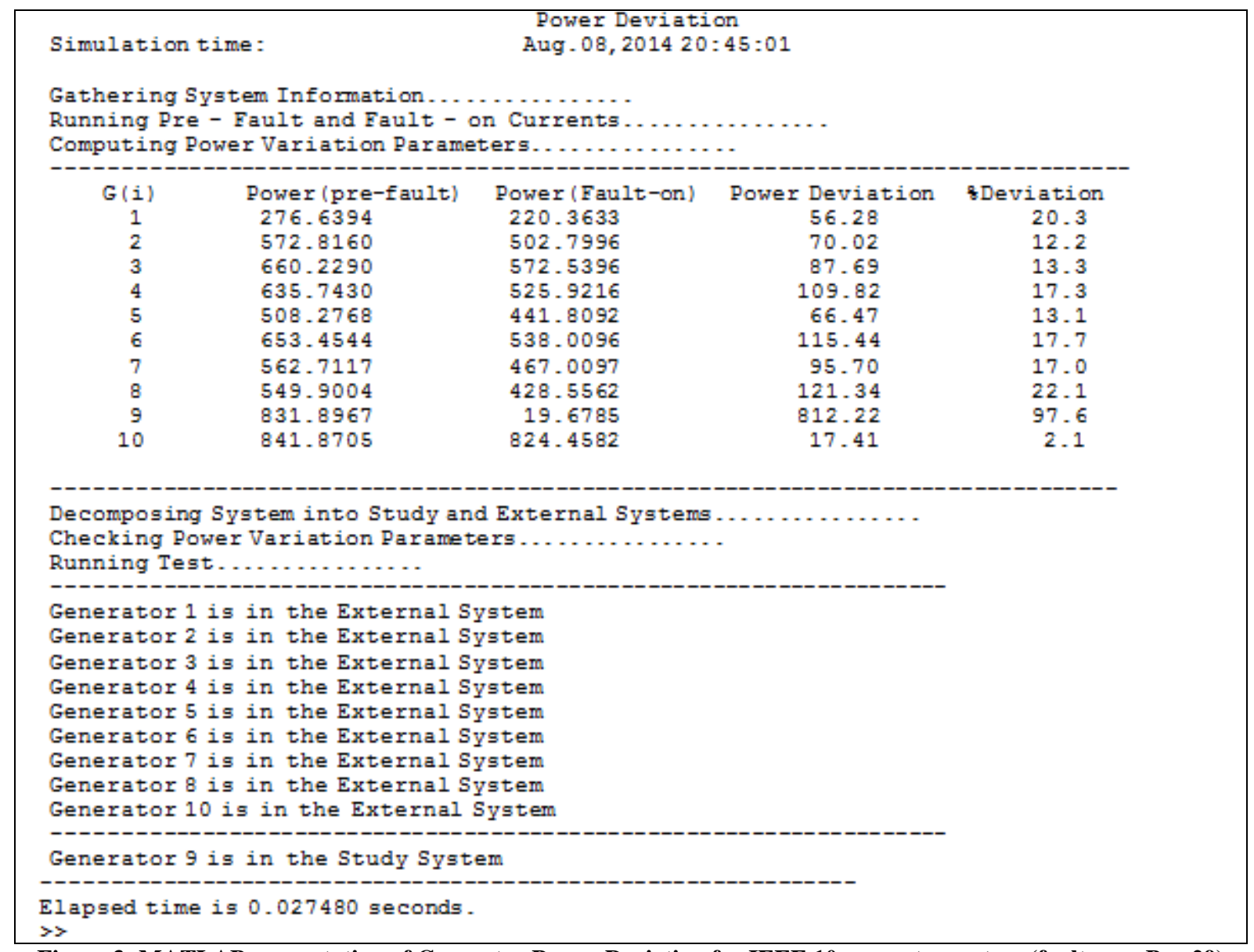

Figure 3: MATLAB computation of Generator Power Deviation for IEEE 10 generator system (fault near Bus 29)

The GPD was calculated for a three-phase fault that occurred at point "P" near Bus 29 of the IEEE 10 generator system and the simulation results are displayed in Figure 3.

It is clear from the GPD results that Generator G9 had the highest percentage of power deviation of $97.6 \%$ and is classified into the study system by the program as shown in Figure 3. This conforms to the results in [3] and also, is realistic because G9 is closest to the point of fault (Bus 29). It is also seen that G8 which appears closest to G9 by inspection, experienced a higher power deviation than other generators. We should also consider that other machine and network characteristics could affect power deviation.

\subsubsection{ADI}

The ADI (pre-fault) was obtained using a MATLAB script, and is shown in the Figure 4. For illustrative purposes, examining Figure 4 and checking generator 8's (G8) closeness to other generators. One would find that only G1, G9 and G10 are reasonably close to G8 because these satisfy the electrical coupling criteria of eqn. (8), $\alpha_{i k} \geq 1.0$ (i.e. $\alpha_{8,1}=$ 2.7127; $\left.\alpha_{8,9}=1.1415 ; \alpha_{8,10}=2.3239\right)$. The remaining generators are electrically distant based on this criterion and this does not qualify them to be considered candidates for coherency testing with G8. This also appears realistic when compared to the physical network structure of Figure 1.

However, in the post-fault state shown in Figure 5, after line $26-29$ has been tripped, the electrical closeness between G8 and G9 is lost as $\left(\alpha_{8,9}=0.8862\right)$ the criterion of eqn. (8) is no longer met. From the network diagram of Figure 1, it can also be seen that the link between G8 and G9 is weaker after the tripping of line $26-29$, causing a reduction in admittance.

These illustrations show how importantly the ADI could lead to deeper understanding of the structure of the transmission network and could give a system planner or operator, insight to possible security threats.

\subsubsection{CI-Inertia and damping}

The CI (inertia and damping) were computed using scripts (B index.m and $\mathrm{Y}$ index.m) and displayed in Figure 6 and 7 respectively. The MATLAB scripts implements eqn. (14) and (15) and the resulting CI are symmetric matrices. The MATLAB tri 1 function was used to obtain the lower triangular part of the symmetric matrices. The programs could be invoked using the following commands: $>>b_{\text {_ index }}$

$>>_{\text {y_index }}$

The following pseudo code could be used to obtain the inertia index:

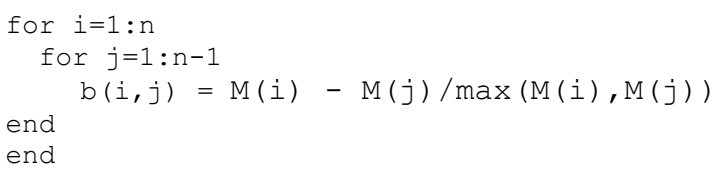

The pseudo code for the damping index is very similar to that of inertia, the only difference being that it calculates eqn. (15). 
By inspecting the three indices of Figure 5, 6 and 7, the coherent generators were identified using eqn. (8) and the coherency criteria. The coherent groups are:

Coherent Group I: 2 and 3

Coherent Group II: $\quad$ 4, 6 and 7

When the $\gamma$-index is neglected in the coherency criteria, generators 1 and 8 form a coherent group and generator 5 is part of Group II. The three indices and the coherent groups obtained are in conformity with the results obtained in [3].

\subsection{DE Computation}

\subsubsection{IEEE 39-bus Test Case}

The coherent machines obtained from the last section were aggregated using the simulation program. The equivalent generator parameters for Coherent Group II are obtained from the simulation program as shown in Figure 8. These results are in conformity to those obtained in [3] for the same fault condition. The DE script (Dynagg.m) is invoked by the following command:

$>$ dynagg

\subsubsection{Nigeria Power System Case Sample}

To compare the rotor angle variations of coherent generators with their DEs, a three-phase fault was initiated near Bus 10, tripping line $10-27$ of the Nigeria power system. The power deviation of all machines during this disturbance was obtained, and the generator at Bus 4 (which was closest to the fault location) experienced the highest deviation $(75.4 \%)$ followed by the generator at Bus 6 (25.9\%).

\begin{tabular}{|c|c|c|c|c|c|c|c|c|c|}
\hline \multicolumn{9}{|c|}{$\begin{array}{c}\text { Admittance Distance-INDEX (Pre-Fault Condition) } \\
\text { Aug . 08,2014 21:00:19 }\end{array}$} & \\
\hline 1 & 2 & 3 & 4 & 5 & 6 & 7 & 8 & 9 & 10 \\
\hline 1.0000 & 0.5366 & 0.6899 & 0.6957 & 0.2409 & 0.6374 & 0.5425 & 1.7896 & 0.9496 & 2.9248 \\
\hline 0.9335 & 1.0000 & 1.8019 & 0.6603 & 0.2286 & 0.6049 & 0.5149 & 0.5231 & 0.4310 & 2.7735 \\
\hline 1.0097 & 1.5160 & 1.0000 & 0.7903 & 0.2736 & 0.7240 & 0.6162 & 0.5708 & 0.4861 & 2.5267 \\
\hline 0.9240 & 0.5042 & 0.7171 & 1.0000 & 1.5650 & 1.3535 & 1.1520 & 0.5694 & 0.6339 & 1.2403 \\
\hline 0.6787 & 0.3703 & 0.5267 & 3.3199 & 1.0000 & 0.9942 & 0.8462 & 0.4182 & 0.4656 & 0.9110 \\
\hline 0.8470 & 0.4621 & 0.6574 & 1.3544 & 0.4689 & 1.0000 & 2.6158 & 0.5219 & 0.5810 & 1.1369 \\
\hline 0.8018 & 0.4375 & 0.6223 & 1.2822 & 0.4439 & 2.9095 & 1.0000 & 0.4941 & 0.5501 & 1.0763 \\
\hline 2.7127 & 0.4558 & 0.5912 & 0.6499 & 0.2250 & 0.5953 & 0.5067 & 1.0000 & 1.1415 & 2.3239 \\
\hline 1.8289 & 0.4773 & 0.6397 & 0.9193 & 0.3183 & 0.8421 & 0.7168 & 1.4505 & 1.0000 & 1.7828 \\
\hline 2.2179 & 1.2090 & 1.3092 & 0.7082 & 0.2452 & 0.6488 & 0.5522 & 1.1626 & 0.7019 & 1.0000 \\
\hline
\end{tabular}

Figure 4: MATLAB computation of $\alpha$ - index (pre-fault) for IEEE 10 generator system

\begin{tabular}{|c|c|c|c|c|c|c|c|c|c|}
\hline $\begin{array}{l}\text { Simulation } \\
\text { Enter the N } \\
\text { Enter the M }\end{array}$ & $\begin{array}{l}\text { ime: } \\
\text { ber of } \mathrm{M} \\
\text { hine No }\end{array}$ & $\begin{array}{l}\text { Admitt } \\
\text { aines in } \\
\text { the Ge }\end{array}$ & $\begin{array}{l}\text { Aug } 08 \\
\text { he Exter } \\
\text { rators i }\end{array}$ & $\begin{array}{l}\text { :e-INDEX } \\
01421: 0 \\
\text { the Stud Area } \\
\text { the Sto }\end{array}$ & $\begin{array}{l}\text { ost-Fau } \\
49 \\
9\end{array}$ & Conditi & $-->9$ & & \\
\hline 1 & 2 & 3 & 4 & 5 & 6 & 7 & 8 & 9 & 10 \\
\hline 1.0000 & 0.5366 & 0.6902 & 0.6995 & 0.2422 & 0.6408 & 0.5454 & 1.7877 & 0.7394 & 2.9142 \\
\hline 0.9385 & 1.0000 & 1.7941 & 0.6617 & 0.2291 & 0.6062 & 0.5159 & 0.5284 & 0.3374 & 2.7664 \\
\hline 1.0149 & 1.5084 & 1.0000 & 0.7909 & 0.2739 & 0.7246 & 0.6167 & 0.5764 & 0.3803 & 2.5210 \\
\hline 0.9316 & 0.5039 & 0.7164 & 1.0000 & 1.5538 & 1.3490 & 1.1482 & 0.5767 & 0.4950 & 1.2448 \\
\hline 0.6855 & 0.3708 & 0.5272 & 3.3023 & 1.0000 & 0.9927 & 0.8449 & 0.4244 & 0.3642 & 0.9160 \\
\hline 0.8545 & 0.4622 & 0.6572 & 1.3507 & 0.4677 & 1.0000 & 2.5999 & 0.5290 & 0.4540 & 1.1418 \\
\hline 0.8092 & 0.4377 & 0.6223 & 1.2791 & 0.4429 & 2.8928 & 1.0000 & 0.5010 & 0.4299 & 1.0813 \\
\hline 2.7015 & 0.4566 & 0.5925 & 0.6544 & 0.2266 & 0.5995 & 0.5102 & 1.0000 & 0.8862 & 2.3178 \\
\hline 1.9105 & 0.4985 & 0.6683 & 0.9603 & 0.3325 & 0.8797 & 0.7487 & 1.5152 & 1.0000 & 1.8624 \\
\hline 2.2164 & 1.2031 & 1.3040 & 0.7108 & 0.2461 & 0.6512 & 0.5543 & 1.1665 & 0.5482 & 1.0000 \\
\hline
\end{tabular}

Figure 5: MATLAB computation of $\alpha$ - index (post-fault) for IEEE 10 generator system 


\begin{tabular}{|c|c|c|c|c|c|c|c|c|}
\hline \multicolumn{9}{|c|}{ 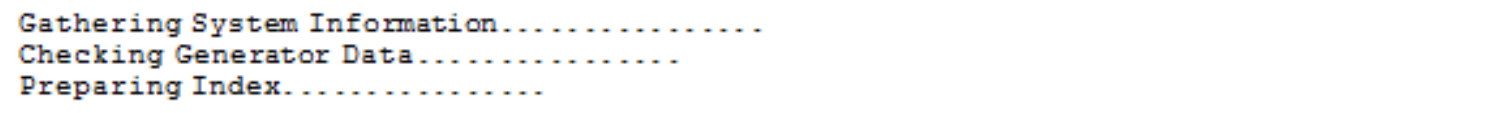 } \\
\hline \multicolumn{9}{|c|}{ The B - Index (Inertia Condition is $\ldots . . . . . .$. ) } \\
\hline 0 & 0 & 0 & 0 & 0 & 0 & 0 & 0 & 0 \\
\hline 0.2786 & 0 & 0 & 0 & 0 & 0 & 0 & 0 & 0 \\
\hline 0.1476 & 0.1536 & 0 & 0 & 0 & 0 & 0 & 0 & 0 \\
\hline 0.3190 & 0.0561 & 0.2011 & 0 & 0 & 0 & 0 & 0 & 0 \\
\hline 0.3810 & 0.1419 & 0.2737 & 0.0909 & 0 & 0 & 0 & 0 & 0 \\
\hline 0.1714 & 0.1293 & 0.0279 & 0.1782 & 0.2529 & 0 & 0 & 0 & 0 \\
\hline 0.3714 & 0.1287 & 0.2626 & 0.0769 & 0.0152 & 0.2414 & 0 & 0 & 0 \\
\hline 0.4214 & 0.1980 & 0.3212 & 0.1503 & 0.0654 & 0.3017 & 0.0795 & 0 & 0 \\
\hline 0.1786 & 0.1217 & 0.0363 & 0.1710 & 0.2464 & 0.0086 & 0.2348 & 0.2957 & 0 \\
\hline 0.9160 & 0.9394 & 0.9284 & 0.9428 & 0.9480 & 0.9304 & 0.9472 & 0.9514 & 0.9310 \\
\hline
\end{tabular}

Figure 6: MATLAB computation of $\beta$ - index for IEEE 10 generator system

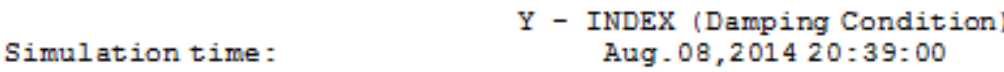

$\begin{array}{ccccccccc}0 & 0 & 0 & 0 & 0 & 0 & 0 & 0 \\ 0.7047 & 0 & 0 & 0 & 0 & 0 & 0 & 0 & 0 \\ 0.6590 & 0.1340 & 0 & 0 & 0 & 0 & 0 & 0 \\ 0.7276 & 0.0775 & 0.2011 & 0 & 0 & 0 & 0 & 0 \\ 0.1798 & 0.6400 & 0.5843 & 0.6679 & 0 & 0 & 0 & 0 \\ 0.6686 & 0.1091 & 0.0279 & 0.1782 & 0.5959 & 0 & 0 \\ 0.6857 & 0.0605 & 0.0782 & 0.1333 & 0.6168 & 0.0517 & 0 & 0 \\ 0.7434 & 0.1309 & 0.2474 & 0.0579 & 0.6872 & 0.2258 & 0.1835 & 0 \\ 0.7653 & 0.2051 & 0.3117 & 0.1384 & 0.7139 & 0.2919 & 0.2532 & 0.0854 & 0 \\ 0.7900 & 0.9380 & 0.9284 & 0.9428 & 0.8278 & 0.9304 & 0.9340 & 0.9461 & 0.9507\end{array}$

Figure 7: MATLAB computation of $\gamma$-index for IEEE 10 generator system

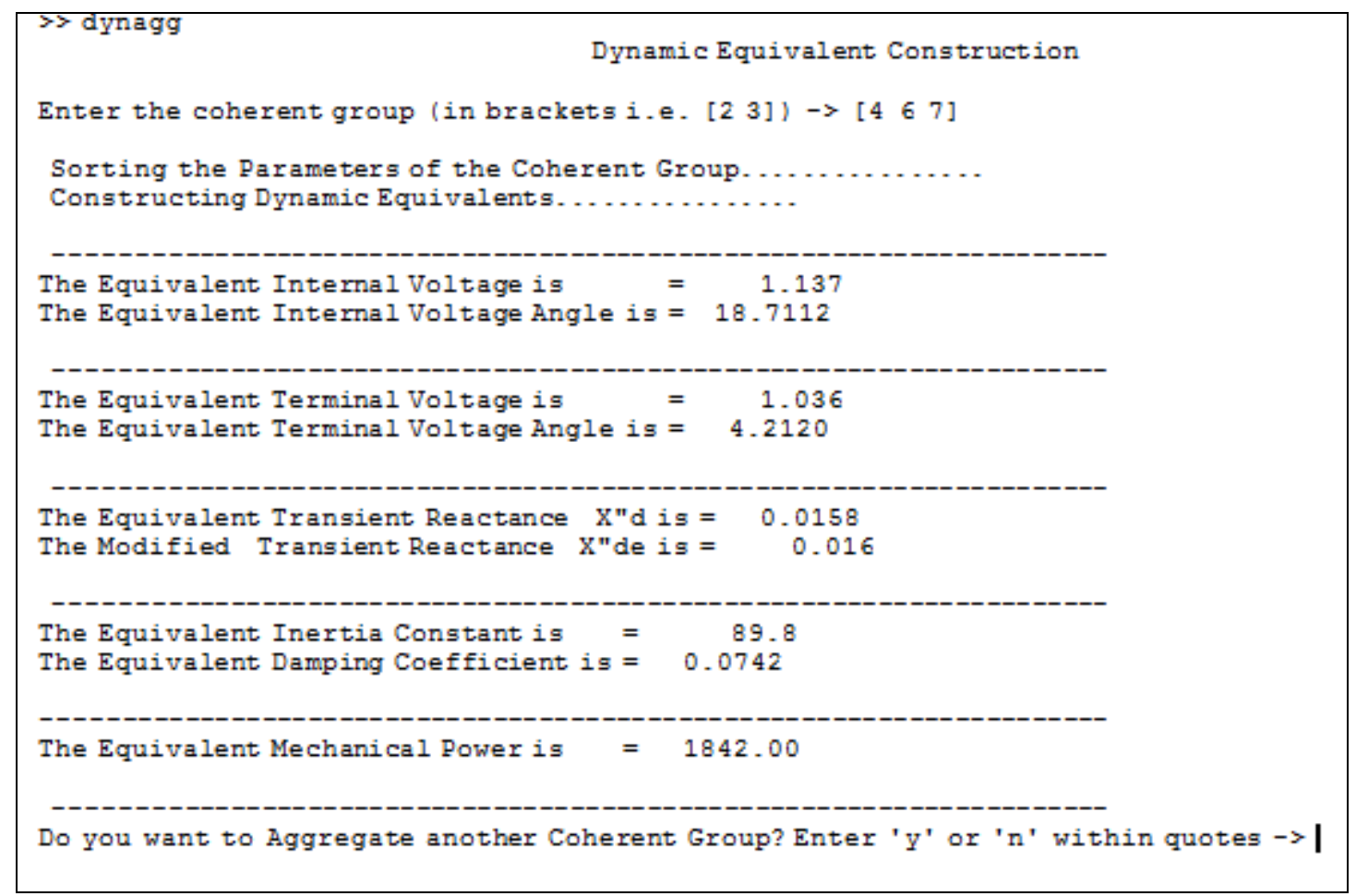

Figure 8: MATLAB computation to construct a DE for Coherent Group II $(4,6$ and 7$)$ in the IEEE 10 generator system 
The computed coherency indices were used to identify two groups of coherent generators for the Nigeria power system below:

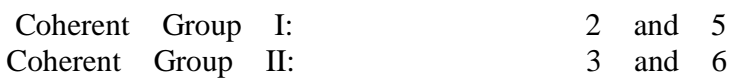

A DE for each of these generator groups was computed, reconnected into the network and their parameters were used to obtain the direct simulation for the same fault location.

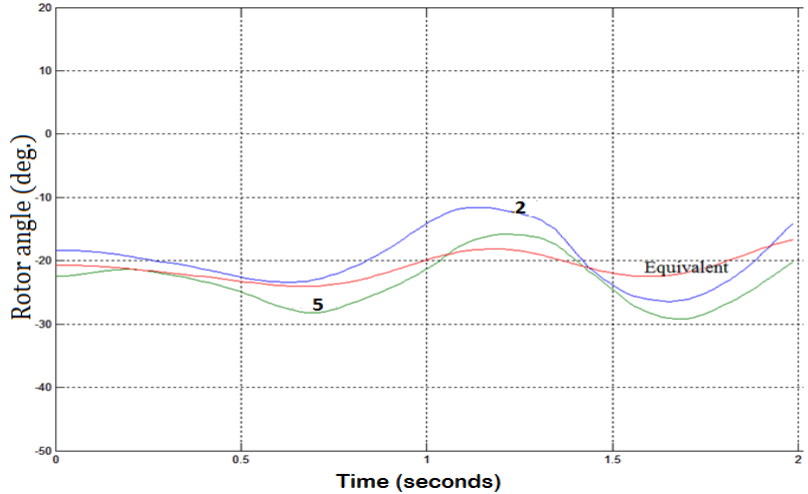

Figure 9: Rotor angle variations vs Time plot of the coherent generators and their dynamic equivalents (Nigeria power system test case)

\section{CONCLUSION}

In this paper, we have shared a simulation and relevant computer methods for power system dynamic equivalents based on coherent generators. The features of the simulation package include:

- Computation of power deviation of all generators during a large disturbance and decomposition of the power system into study and remote regions.

- Simulation of admittance distance for identifying electrically close generators.

- Calculation of Coherency Indices (inertia and damping)

- Construction of dynamic equivalents for coherent generators.

The simulation results for coherency parameters and dynamic equivalents are validated by comparison of the IEEE 39-bus test case with those in existing literature. The application of DE to transient stability analysis of the Nigeria power system is validated by comparing the direct simulations of the coherent generators with their dynamic equivalents.

\section{ACKNOWLEDGMENTS}

Many thanks to academics who have contributed immensely to research in dynamic equivalents.

\section{REFERENCES}

[1] Milano F. 2005 An open source power system analysis toolbox. IEEE Trans. Power Syst., 20(3): 1199-1206.

[2] J. Chow and K. Cheung, 1992 A toolbox for power system dynamics and control engineering education and research. IEEE Transactions on Power Systems, vol. 7, no. 4, pp. 1559-1564.

[3] Guruprasada V., Rau, and Yeakub Hussain MD., 1994 Coherent Generators. Allied Publishers Limited, New Delhi.

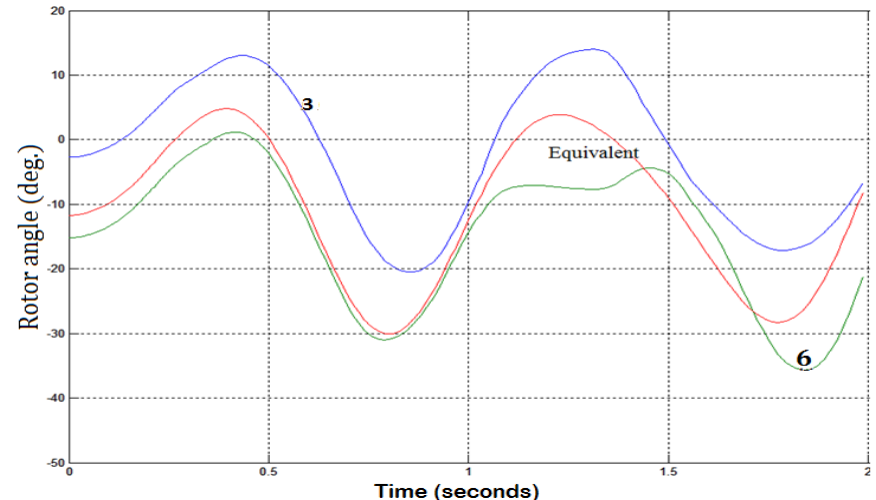

Figure 9 shows a comparison of the rotor angle variations of the coherent generators (in the full system) along with their DEs (from the reduced system) for the same fault location. The direct simulation lasted for 2 seconds. It is obvious that the group's equivalent generator's rotor angle variation approximates those of the coherent generators.

After obtaining dynamic equivalents, the number of generators in the system was reduced from 9 to 7 . This is the same with the number of differential equations to be solved.

[4] J., Arrillaga and C.P., Arnold, 1990 Computer Analysis of Power Systems. John Willy and Sons Inc, New York.

[5] A., Monticelli, 1999 State Estimation in Electric Power Systems. Kluwer. Academic Publishers, Norwell, USA.

[6] Hadi Saadat. 1999 Power System Analysis. Tata McGraw- Hill Publishing Company Ltd., New Delhi.

[7] K.W Chan., R.W., Dunn, A.R., Daniels, P.H., Buxton, A.O., Ekwue and M.J. Rawlings. On-line Dynamic Security Contingency Screening and Ranking. Proc. of the IEEE-Generation, Transmission and Distribution, Vol. 144 no 2, March 1997, pp 132-138.

[8] R. D. Zimmerman, C. E. Murrillo-Sanchez, and D. Gan, 2005. Matpower,Version 3.0.0, User's Manual, Power System Engineering Research Center, Cornell University.

[9] Kimbark E.W., 1948 Power System Stability, Vol. 1, John Willy and Sons Inc, New York.

[10] Elgerd, O. I. 1983 Electric Energy System Theory", Tata McGraw-Hill Publishing Company Ltd., New Delhi.

[11] J. J., Grainger, and W .D. Stevenson, Jr.,"Power System Analysis", McGraw-Hill Inc, Singapore, 1994.

[12] IEEE 10 generator 39 bus system http://www.sel.eesc.usp.br/ieee/IEEE39/main.htm. Accessed: August, 2014

[13] Erlich, I., Kasztel, Z., Schegner, P., 2002 Enhanced Model Based Technique for Construction of Power System Dynamic Equivalents, $14^{\text {th }}$ PSCC, Sevilla, Session 02,Paper 2, 24-28, pp 1-7.

[14] P., Neriman and E., Atmaca, 2002 A Correlation Based Approach for Power Network Reduction. Ystanbul Unyversytey Mahendyslyk, Vol.1, No. 1, pp 64-76. 